\title{
MicroRNA-Mediated Direct Reprogramming of Human Adult Fibroblasts Toward Cardiac Phenotype
}

\author{
C. Paoletti ${ }^{1}$, C. Divieto ${ }^{2}$, G. Tarricone', F. Di Meglio ${ }^{3}$, D. Nurzynska ${ }^{3}$ and V. Chiono ${ }^{1 *}$
}

${ }^{1}$ Department of Mechanical and Aerospace Engineering, Politecnico di Torino, Turin, Italy, ${ }^{2}$ Istituto Nazionale di Ricerca Metrologica, Advanced Materials Metrology and Life Science, Turin, Italy, ${ }^{3}$ Department of Public Health, University of Naples Federico II, Naples, Italy

\section{OPEN ACCESS}

Edited by:

Paolo Madeddu,

University of Bristol, United Kingdom

Reviewed by:

Sveva Bollini,

University of Genoa, Italy

Paola Campagnolo,

University of Surrey, United Kingdom

*Correspondence:

v. Chiono

valeria.chiono@polito.it

Specialty section:

This article was submitted to

Tissue Engineering and Regenerative

Medicine,

a section of the journal

Frontiers in Bioengineering and

Biotechnology

Received: 21 August 2019

Accepted: 04 May 2020

Published: 05 June 2020

Citation:

Paoletti C, Divieto C, Tarricone G,

Di Meglio F, Nurzynska D and

Chiono V (2020) MicroRNA-Mediated

Direct Reprogramming of Human

Adult Fibroblasts Toward Cardiac

Phenotype.

Front. Bioeng. Biotechnol. 8:529.

doi: 10.3389/fbioe.2020.00529
Modulation of microRNA expression holds the promise to achieve direct reprogramming of fibroblasts into cardiomyocyte-like cells as a new strategy for myocardial regeneration after ischemic heart disease. Previous reports have shown that murine fibroblasts can be directly reprogrammed into induced cardiomyocytes (iCMs) by transient transfection with four microRNA mimics (miR-1, 133, 208, and 499, termed "miRcombo"). Hence, study on the effect of miRcombo transfection on adult human cardiac fibroblasts (AHCFs) deserves attention in the perspective of a future clinical translation of the approach. In this brief report, we studied for the first time whether miRcombo transient transfection of AHCFs by non-viral vectors might trigger direct reprogramming of AHCFs into cardiomyocyte-like cells. Initially, efficient miRNA delivery to cells was demonstrated through the use of a commercially available transfection agent (DharmaFECT1). Transient transfection of AHCFs with miRcombo was found to upregulate early cardiac transcription factors after 7 days post-transfection and cardiomyocyte specific marker cTnT after 15 days post-transfection, and to downregulate the expression of fibroblast markers at 15 days post-transfection. The percentage of cTnT-positive cells after 15 days from miRcombo transfection was $\sim 11 \%$, as evaluated by flow cytometry. Furthermore, a relevant percentage of miRcombo-transfected AHCFs ( $38 \%)$ displayed spontaneous calcium transients at 30 days post-transfection. Results evidenced the role of miRcombo transfection on triggering the trans differentiation of AHCFs into iCMs. Although further investigations are needed to achieve iCM maturation, early findings from this study pave the way toward new advanced therapies for human cardiac regeneration.

Keywords: cardiac fibroblasts, direct reprogramming, cardiomyocytes, microRNAs, digital droplet PCR (ddPCR)

\section{INTRODUCTION}

Myocardial infarction (MI) remains one of the leading causes of death globally. After MI, the injured area of the heart undergoes a deep tissue remodeling: cardiomyocytes (CMs) death, extracellular matrix (ECM) degradation and increased myocardial fibrosis dramatically impair cardiac function, leading to heart failure (van den Borne et al., 2010). The only available therapeutic 
strategy against heart failure is heart transplantation. Hence, cardiac regeneration is still a major clinical challenge.

An ideal cardiac regenerative medicine strategy should replace lost CMs and recover myocardial contractility. Recently, efforts have been addressed at reducing the extent of cardiac fibrotic scar, as it reduces the contractile heart functionality (Talman and Ruskoaho, 2016). Direct reprogramming of fibroblasts into induced cardiomyocytes (iCMs) represents a new emerging strategy to convert cardiac fibrotic tissue into functional tissue (Bektik and Fu, 2019). Ieda et al. (2010) were the first to report direct reprogramming of mouse fibroblasts into beating iCMs through a simultaneous overexpression of GATA binding protein 4 (GATA4), Myocyte enhancer factor 2C (MEF2C), T-box 5 (TBX5) cardiac transcription factors (TFs) both in vitro and in vivo. Later, Song et al. (2012) added Heart and Neural Crest derivatives expressed 2 (HAND2) to the previous combination of cardiac TFs, increasing reprogramming efficiency of mouse fibroblasts in vitro and improving cardiac function of injured hearts in vivo. Since then, several other direct reprogramming strategies have been reported for mouse and human fibroblasts, generally based on gene therapy via viral overexpression of specific cardiac TFs (Chen et al., 2017).

Efforts are now addressed to develop a direct reprogramming method which is safe and free from ethical and technical concerns associated with genetic manipulation of cells. The use of microRNAs (miRNAs) may represent one valid strategy to induce direct reprogramming of human fibroblasts into iCMs, satisfying such requirements. miRNAs are short noncoding RNAs (of approximately 22 nucleotides) that regulate gene expression post-transcriptionally (He and Hannon, 2004). As cardiac tissue-specific miRNAs play pivotal role in cardiac development and functions, the regulation of their expression would aid in generating functional iCMs starting from human fibroblasts (Tian et al., 2017). Endogenous miRNAs can be easily overexpressed or downregulated through the administration of miRNA mimics or miRNA inhibitors, respectively (Peng et al., 2015). MiRNA mimics and inhibitors hold a great potential as therapeutic agents because of their cytoplasmic activity, relatively small size and ability to be administered systemically or locally by nanoparticle-based delivery systems, avoiding the use of viral vectors (Lee et al., 2019). Modulation of miRNAs for direct reprogramming was first proposed by Jayawardena et al. (2012), who demonstrated that a combination of four miRNA mimics (referred to as "miRcombo": miR-1, 133, 208 and 499) promotes the direct reprogramming of mouse fibroblasts into iCMs both in vitro and in vivo. The selected miRNAs are highly conserved in cardiac and skeletal tissue and have been found to regulate developmental and physiological processes in the heart (van Rooij et al., 2009; Xu et al., 2011). Furthermore, this specific set of microRNAs was found to be expressed in human embryonic stem cells-derived CMs, whereas their expression was nearly undetectable in fibroblasts (Kitchener et al., 2010).

Although the potentiality of miRcombo in reprogramming mouse fibroblasts into iCMs has been demonstrated (Jayawardena et al., 2012, 2015), the translation of miRcombomediated direct reprogramming from mouse to human cells is not straightforward and deserves further investigation. Indeed, human adult fibroblasts are intrinsically more resistant to direct reprogramming than mouse fibroblasts, as demonstrated by those direct reprogramming strategies, first developed on mouse fibroblasts, which failed on human fibroblasts or required substantial adjustments (Ieda et al., 2010; Nam et al., 2013).

In this brief research report, we sought to determine if miRcombo transient transfection of AHCFs was able to initiate the trans-differentiation process of AHCFs toward cardiomyocyte-like cells. After initial assessment of efficient miRNA delivery to AHCFs, cells were transfected with miRcombo, showing increased expression of cardiomyocyte markers and down-regulated expression of fibroblast markers after 7-15 days post-transfection. Moreover, miRcombo treated cells exhibited spontaneous calcium oscillation after 30 days from transfection.

Early findings presented in this brief research report pave the way toward future new advanced approaches for human cardiac regeneration, through in situ direct reprogramming of cardiac fibroblasts populating post-infarct scar into cardiomyocytelike cells.

\section{MATERIALS AND METHODS}

\section{Cell Culture}

Normal human atrial cardiac fibroblasts (AHCFs) were purchased from Lonza (CC-2903; batch number: 0000662121; donor characteristics: male; 48 years old; passage number: 2) and maintained in culture using Fibroblasts Growth Medium-3 (Lonza, CC-4526) containing 10\% fetal bovine serum (FBS), 1\% insulin, 1\% human basal fibroblast growth factor (hFGF-B) and $1 \%$ gentamicin. Cells were expanded until passage four and then used for experiments.

\section{MiRNA Transient Transfection}

AHCFs were plated either in six-well plates at $10 \times 10^{5}$ cells/well (for RNA isolation and flow cytometry experiments), or in $35 \mathrm{~mm}$ $\mu$-dish (Ibidi) at $9 \times 10^{5}$ cells/well (for calcium transient analysis) in Dulbecco's Modified Eagle Medium (DMEM) High Glucose (Gibco) with 10\% FBS (Sigma-Aldrich) and 1\% glutamine (Sigma-Aldrich) 1 day before transfection.

To initially study miRNA internalization and mRNA target downregulation by AHCFs, cells were transfected using DharmaFECT1 (Dharmacon) with miR-1 (miR-1-3p, mirVana miRNA mimic, Life Technologies) or negmiR (Negative Control \#1, mirVana miRNA Mimic, Life Technologies), at a final concentration of $25 \mathrm{nmol} / \mathrm{L}$ according to manufacturer's instructions, in DMEM High Glucose with 10\% FBS and $1 \%$ L-glutamine. After $24 \mathrm{~h}$, the medium was replaced with DMEM High Glucose with 15\% FBS, 1\% glutamine and 1\% penicillin/streptomyocin/ampicillin (Lonza). Culture was continued up to $48 \mathrm{~h}$.

Then, AHCFs were transfected using DharmaFECT1 with miRcombo (miR-1-3p, miR-133a-3p, miR-208a-3p, and miR-499a-5p, mirVana miRNA mimic, Life Technologies) or negmiR (Negative Control \#1, mirVana miRNA Mimic, Life Technologies), at a final concentration of $25 \mathrm{nmol} / \mathrm{L}$ according 
to manufacturer's instructions, in DMEM High Glucose (Gibco) with $10 \%$ FBS and $1 \%$ glutamine. After $24 \mathrm{~h}$, medium was replaced with DMEM High Glucose with 10\% FBS, 1\% glutamine and $1 \%$ penicillin/streptomyocin/ampicillin (Lonza). Culture was continued up to 30 days.

\section{RNA Isolation and Droplet Digital PCR}

Total RNA was extracted using QIAzol Lysis Reagent (Qiagen) according to manufacturer's instructions. RNA concentration and quality were assessed using NanoQuant plate (Tecan Group Ltd). cDNA (200 ng) was obtained using High Capacity cDNA Reverse Transcription Kit (Applied Biosystems).

To study miR-1 uptake by cells, miRNA reverse-transcription PCR was performed using miRCURY LNA RT Kit (Qiagen) at $24 \mathrm{~h}$ (i.e., immediately after transfection) and $48 \mathrm{~h}$ (i.e., at $24 \mathrm{~h}$ from transfection) culture times. RNA samples were diluted to $5 \mathrm{ng} / \mu \mathrm{l}$ in nuclease-free water and the reaction was prepared following the manufacturer's instructions. Droplet digital PCR (ddPCR, Bio-Rad Laboratories) was performed to assess the expression of miR-1 (Hsa-miR-1-3p miRCURY LNA miRNA PCR Assay) using EvaGreen supermix (Bio-Rad Laboratories). Droplet generation was performed according to manufacturer's instructions. Thermal-cycling conditions were: $95^{\circ} \mathrm{C}$ for $5 \mathrm{~min}(1 \mathrm{cycle}), 95^{\circ} \mathrm{C}$ for $30 \mathrm{~s}$ and $55^{\circ} \mathrm{C}$ for $1 \mathrm{~min}$ (40 cycles), $90^{\circ} \mathrm{C}$ for $5 \mathrm{~min}$ ( 1 cycle), and a $4^{\circ} \mathrm{C}$ infinite hold. PCR plate was loaded on Bio-Rad QX100 droplet reader for quantification of cDNA copies/ $\mu \mathrm{L}$. Analysis of the ddPCR data was performed by QuantaSoft analysis software (Bio-Rad Laboratories). No template control with water was included in each assay. Experiments were performed in triplicate and repeated three times.

Downregulation of TWF-1 target after miR-1 transfection was analyzed at $24 \mathrm{~h}$ (i.e., immediately after transfection) and $48 \mathrm{~h}$ (i.e., at $24 \mathrm{~h}$ from transfection) culture times. The expression of TWF-1 (ID assay: dHsaCPE5028122) was analyzed by ddPCR, using ddPCR supermix for probes without dUTP.

Similarly, after miRcombo or negmiR transfection, the expression of GATA4 (ID assay: dHsaCPE5050488), MEF2C (ID assay: dHsaCPE5050696), TBX5 (ID assay: dHsaCPE5048560), HAND2 (ID assay: dHsaCPE5049426) and NKX2.5 (ID assay: dHsaCPE5042098) was evaluated at 7 days post-transfection, while the expression of TNNT2 (ID assay: dHsaCPE5052344), TNNI3 (ID assay: dHsaCPE5050934), VIM (ID assay: dHsaCPE5032314), DDR2 (ID assay: dHsaCPE5048156) and FSP-1 (ID assay: dHsaCPE5042942) was assessed at 15 days post-transfection, using ddPCR supermix for probes without dUTP. Glyceraldehyde 3-phosphate dehydrogenase (GAPDH; ID assay: dHsaCPE5031597) was used as a housekeeping gene to perform quantitative normalization.

For TWF-1 and cardiac markers analysis, droplet generation was performed according to manufacturer's instructions. Thermal-cycling conditions were $95^{\circ} \mathrm{C}$ for $10 \mathrm{~min}$ ( 1 cycle), $94^{\circ} \mathrm{C}$ for $30 \mathrm{~s}$ and $55^{\circ} \mathrm{C}$ for $30 \mathrm{~s}$ ( 40 cycles), $98^{\circ} \mathrm{C}$ for $10 \mathrm{~min}$ ( 1 cycle), and a $4^{\circ} \mathrm{C}$ infinite hold.

In all ddPCR experiments, PCR plate was loaded on Bio-Rad QX100 droplet reader for quantification of cDNA copies/ $\mu \mathrm{L}$. Analysis of the ddPCR data was performed by QuantaSoft analysis software (Bio-Rad Laboratories). No template control with $\mathrm{H}_{2} \mathrm{O}$ was included in each assay. Experiments were performed in triplicate and repeated three times.

Results are reported as the concentration (cDNA copies/ $\mu \mathrm{L})$ of the gene of interest calculated on the concentration mean (cDNA copies $/ \mu \mathrm{L}$ ) of GAPDH.

\section{Flow Cytometry}

After transfection with miRcombo or negmiR, AHCFs were cultured for additional 15 days. Then, cells were trypsinized with $0.05 \%$ Trypsin/EDTA (Sigma Aldrich) and permeabilized with $0.5 \% \mathrm{v} / \mathrm{v}$ Tween 20 in phosphate buffered saline (PBS) for 5 min. Ice cold PBS with $10 \%$ FBS and 1\% sodium azide (Sigma Aldrich) was used for washing between each step. Cells were incubated with Cardiac Troponin $\mathrm{T}$ primary antibody (cat \#701620, Invitrogen) for $1 \mathrm{~h}$ at $4^{\circ} \mathrm{C}$ and Alexa Fluor 488conjugated secondary antibody (ab150077, Abcam) for $1 \mathrm{~h}$ at $4^{\circ} \mathrm{C}$ in the dark. Cells were run on Guava EasyCyte (Merck) flow cytometer and data analysis was performed using GuavaSoft 3.2.

\section{Measurements of Spontaneous Calcium Transients}

Calcium transient was analyzed in AHCFs after 30 days posttransfection with miRcombo or negmiR. To record calcium transients, cells were loaded with $5 \mu \mathrm{M}$ of Fluo- $4 \mathrm{AM}$ (Invitrogen) in modified Tyrode's solution $(140 \mathrm{mM} \mathrm{NaCl}, 5 \mathrm{mM} \mathrm{KCl}$, $1.8 \mathrm{mM} \mathrm{CaCl}_{2}, 1 \mathrm{mM} \mathrm{MgCl}_{2}, 10 \mathrm{mM}$ glucose and $10 \mathrm{mM}$ Hepes) with $0.1 \%$ bovine serum albumin (BSA) at $37^{\circ} \mathrm{C}$ for 20 min while shielded from light. Cells were washed in modified Tyrode's solution at $37^{\circ} \mathrm{C}$ for $10 \mathrm{~min}$. Calcium transient was recorded using Nikon Eclipse Ti2 spinning disk and NISElements software (Nikon). At least 4 fields were observed for each sample. Data were analyzed using ImageJ software (NIH) and $\mathrm{Ca}^{2+}$ level was reported as $\mathrm{F} / \mathrm{F}_{0}$ ratio, where $\mathrm{F}$ is the intensity of fluorescence emission recorded for each cell, while $F_{0}$ is the background fluorescence.

\section{Statistical Analysis}

The results are shown as means \pm standard error of the mean (SEM) of replicate experiments. Statistical analyses were performed with Student's $t$-test, with $\mathrm{p}$-value reported as ${ }^{*} p<0.05$ considered statistically significant, ${ }^{* *} p<0.01$ considered highly significant and ${ }^{* *} p<0.0001$ very highly significant. All graphs were prepared using GraphPad software.

\section{RESULTS}

We initially studied the efficiency of DharmaFECT1 in delivering miRNAs to AHCFs, using miR-1 as a model miRNA, as compared to a non-targeting miRNA negative control (negmiR). DdPCR analysis of miR-1 transfected cells showed relative overexpression of miR-1, and knockdown of TWF-1 target gene in AHCFs, at 24 and 48 h culture times (Figures 1A,B).

Then, the effect of miRcombo transient transfection on AHCFs trans-differentiation into iCMs was evaluated at different 
A

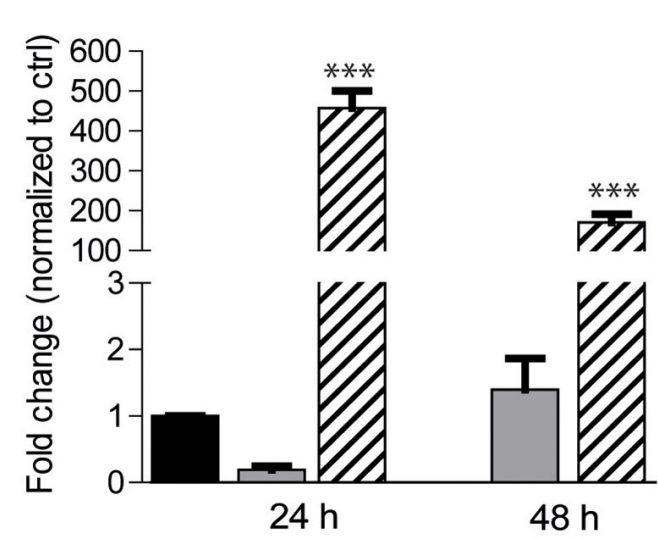

B

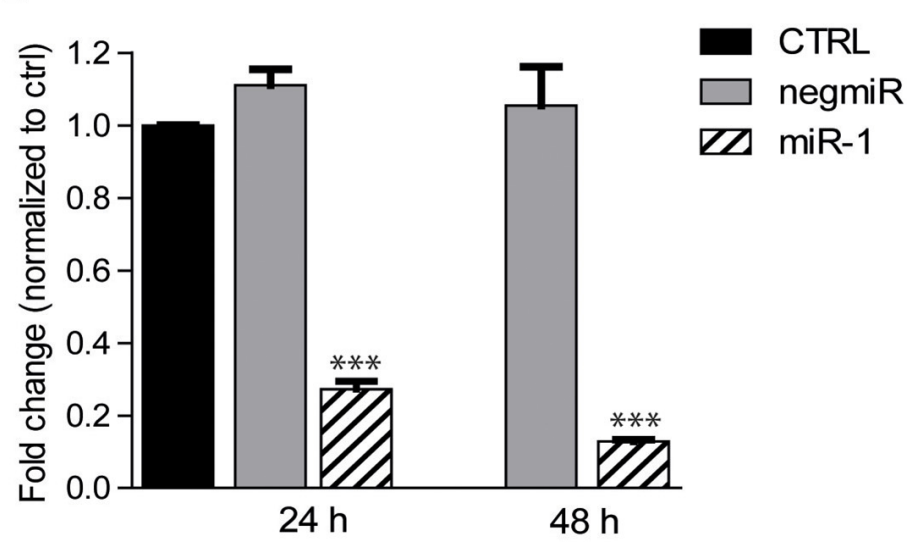

FIGURE 1 | DharmaFECT1 mediated delivery of miR-1 and TWF-1 target downregulation in AHCFs. (A) Fold change of miR-1 expression (relative to untransfected control) in AHCFs transfected with negmiR or miR-1 using DharmaFECT1 at $24 \mathrm{~h}$ (i.e., immediately post-transfection) and $48 \mathrm{~h}$ (i.e., $24 \mathrm{~h}$ post-transfection) culture times, analyzed by ddPCR. Data are representative of three independent experiments. Stated $p$-value ${ }^{* * *} p<0,001$ is reported versus negmiR control. (B) Fold change of TWF-1 mRNA target expression (relative to untransfected control) in AHCFs transfected with negmiR or miR-1 using DharmaFECT1 at 24 h (i.e., immediately post-transfection) and $48 \mathrm{~h}$ (i.e., $24 \mathrm{~h}$ post-transfection) culture times. Data are representative of three independent experiments. Stated $p$-value ${ }^{* * *} p<0,001$ is reported versus negmiR control. CTRL, untransfected control.

time points by different analyses depicted in Figure 2A. As a negative control, AHCFs were also transfected with negmiR.

DdPCR analysis showed that miRcombo transfected cells upregulated the expression of early cardiac transcription factors (TFs), with a significantly increased expression of GATA4 $(p=0.007), \operatorname{MEF} 2 C(p=0.0004)$, TBX5 $(p<0.0001)$ and HAND2 $(p=0.0001)$ cardiac TFs, compared to negmiR transfected cells, 7 days after transfection (Figures 2B-E). Additionally, the expression of NKX2.5 cardiac TF was increased in miRcombo transfected cells compared to controls, although not significantly (Figure 2F).

As a further step, the expression of mature cardiomyocyte markers was evaluated in miRcombo transfected AHCFs at 15 days after transfection. The expression of TNNT2, which encodes for cardiac troponin $\mathrm{T}$ (cTnT), was significantly increased in miRcombo-transfected cells compared to negmiR controls $(p=0.0004)$ (Figure 3A). Also, TNNI3 expression was slightly increased in miRcombo transfected cells compared to controls, although non-significantly (Figure 3B).

The reprogramming efficiency of miRcombo-transfected cells was evaluated using flow cytometry. Notably, approximately $11 \%$ of fibroblasts were $\mathrm{cTnT}^{+}$after 15 days culture time from miRcombo transfection, whereas very low percentage of $\mathrm{cTnT}^{+}$cells $(0.3 \%)$ was measured in negmiR transfected cells (Figure 3C).

Along with the upregulation of cardiomyocyte markers, ddPCR analysis showed that the expression of fibroblast markers, such as vimentin (VIM, $p=0,0003$ ), discoidin domain receptor$2(D D R 2, p=0,0004)$ and fibroblast specific protein-1 (FSP$1 /$ S100A4, $p<0,0001)$ was significantly downregulated in miRcombo-transfected cells compared to controls after 15 days culture time from miRcombo transfection (Figures 3D-F).

Overall, the results suggested that, after 15 days from reprogramming induction, miRcombo-transfected cells started to display functional cardiomyocyte-specific markers along with a reduced fibroblast phenotype.

Finally, to determine whether miRcombo-transfected AHCFs exhibited functional cardiomyocyte properties after a long period of culture, we examined intracellular calcium oscillation at 30 days after transfection. Results showed that miRcombo transfection triggered calcium oscillation, lasting nearly $1 \mathrm{~min}$, in $\sim 38 \%$ of total cells, while rare or no calcium oscillation was found in negmiR transfected control cells (Figures 4A-C).

In conclusion, results demonstrated that miRcombotransfection triggered transdifferentiation of AHCFs into cardiomyocyte-like cells, also increasing functional cardiomyocyte properties compared to AHCFs transfected with negmiR.

\section{DISCUSSION}

Direct reprogramming of human cardiac fibroblasts into cardiomyocytes represents an attractive approach to revert cardiac fibrosis and recover heart function after MI (Chen et al., 2017), avoiding the need for cell-based therapies to replace cardiomyocytes (Hashimoto et al., 2018). Cardiac fibrotic scar is mainly composed by activated fibroblasts (myofibroblasts), while the target healthy cardiac tissue contains 20\% cardiac fibroblasts and 35\% cardiomyocytes (Gray et al., 2018). Ideally, through direct reprogramming, a suitable number of fibroblasts in the scar would be converted into cardiomyocytes to re-establish cell-cell connections and functionality of healthy cardiac tissue.

Since the first evidence of direct reprogramming of fibroblasts into cardiomyocytes in 2010 (Ieda et al., 2010), several efforts have been performed to directly reprogram fibroblasts into iCMs, using combinations of cardiac transcription factors (Song et al., 2012), microRNA mimics (Jayawardena et al., 2012), 


\section{A}

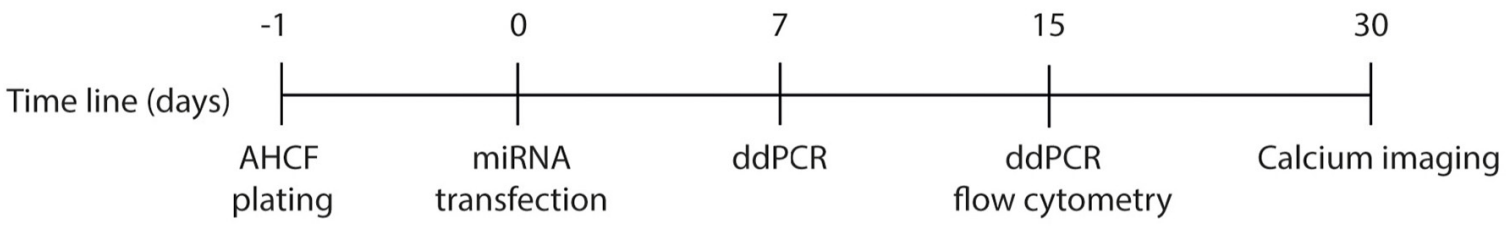

B

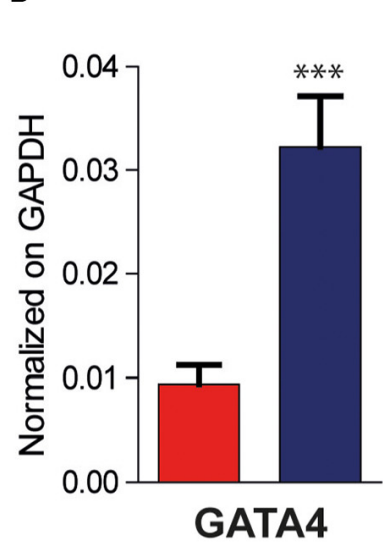

C

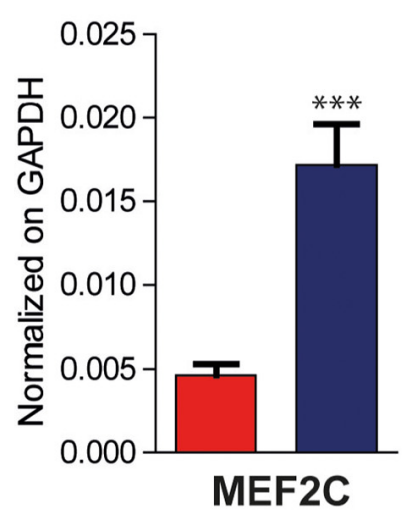

D

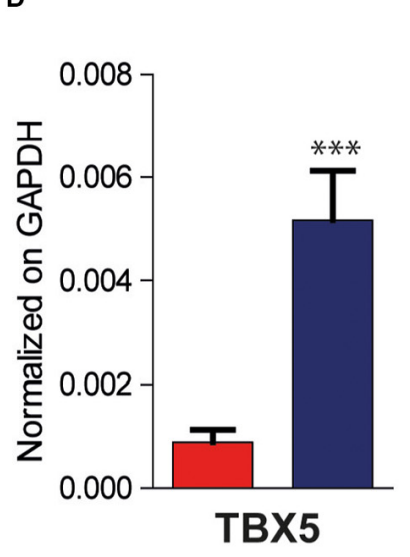

E

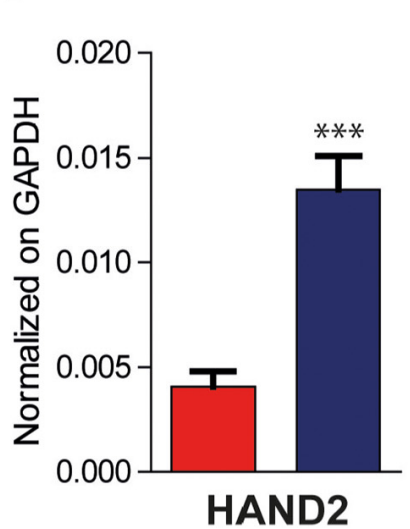

F

$\square$ negmiR $\square$ miRcombo

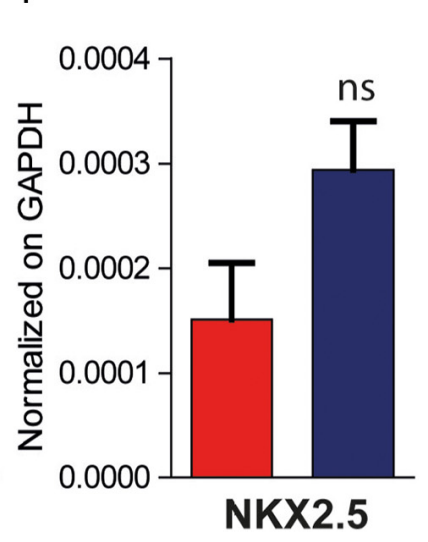

FIGURE 2 | MiRcombo-transfected AHCFs show increased cardiac transcription factor expression. (A) Representative scheme of experimental design. AHCFs were transfected with miRcombo or negative control (negmiR). The acquisition of cardiomyocyte-associated features was evaluated after 7 days (cardiac transcription factors), 15 days (cardiomyocyte and fibroblast markers) and 30 days (calcium transients). (B-F) Gene expression of cardiac transcription factors. The expression of GATA4, MEF2C, TBX5, HAND2, and NKX2.5 was evaluated by ddPCR 7 days post transfection in AHCFs transfected with miRcombo (red) or negmiR (blue). Data are representative of three independent experiments, each performed in triplicate. Stated $P$-value is versus negmiR controls.

and small molecules (Fu et al., 2015). However, most direct reprogramming methods have been generally optimized on mouse cells, which does not assure their efficacy on AHCFs (Chen et al., 2017). Indeed, AHCFs are known to be more resistant to cellular reprogramming than murine fibroblasts due to epigenetic barriers that hinder phenotypic changes (Talkhabi et al., 2017).
Successful direct reprogramming of AHCFs into iCMs was achieved by inducing the expression of cardiac TFs and miRNAs, through retroviruses or inducible lentiviruses (Nam et al., 2013; Muraoka et al., 2014), which cause stable and heritable integration of a specific nucleic acid sequence into the target cell genome (Chen et al., 2017). As an example, Nam et al. (2013) directly reprogrammed 

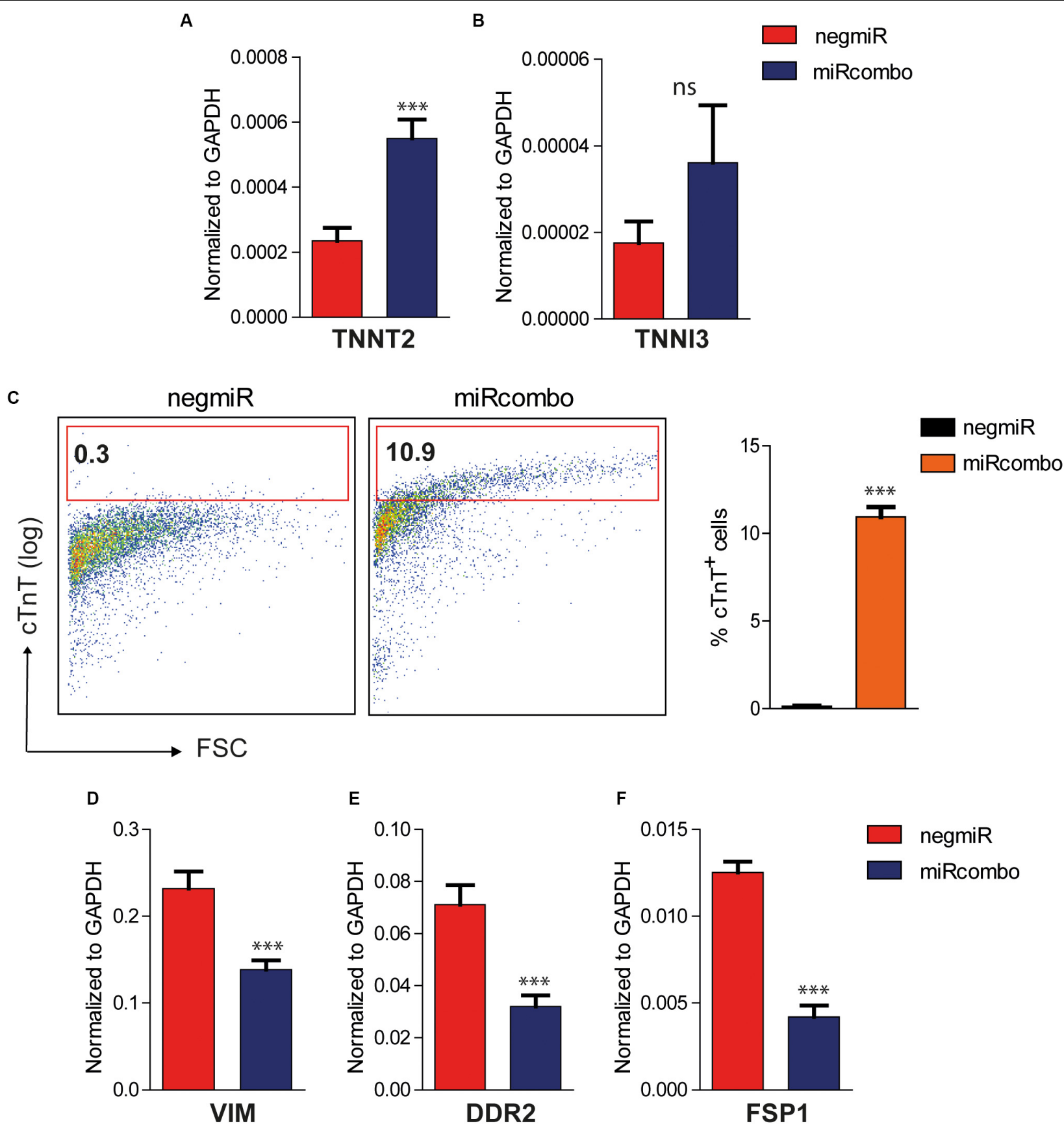

FIGURE 3 | Induction of cardiomyocyte markers in miRcombo-transfected AHCFs. (A,B) Gene expression of cardiomyocyte markers TNNT2 and TNNI3 in AHCFs transfected with miRcombo (red) or negmiR (blue) evaluated by ddPCR. Data are representative of three independent experiments, each performed in triplicate. (C) Representative flow plots (left panel) and percentage (right panel) of cTnT positive cells in AHCFs transfected with miRcombo $(n=3)$ and negmiR $(n=3) 15$ days after transfection. (D-F) Gene expression of fibroblastic markers VIM, DDR2, and FSP-1 in AHCFs transfected with miRcombo (red) or negmiR (blue) by ddPCR. Data are representative of three independent experiments, each performed in triplicate. Stated $P$-value is versus negmiR.

AHCFs into iCMs with an efficiency of $\sim 13 \%$ (measured by $\mathrm{CTnT}^{+}$cells at 2 weeks) by retroviral overexpression of miR-1, miR-133 and specific cardiac TFs. On the other hand, Muraoka et al. (2014) found out $\sim 20 \%$ of $\mathrm{cTnT}^{+}$cells after 1 week from simultaneous transfection of AHCFs with different TFs (by lentiviral vectors) and
miR-133 (by Lipofectamine 2000), however, they used relatively young AHCFs (average age: 35 years old) and did not analyse cell functional properties. Overall, although effective, viral vectors suffer from potential risks which currently hinder an in situ clinical application (Labatut and Mattheolabakis, 2018). 


\section{A}

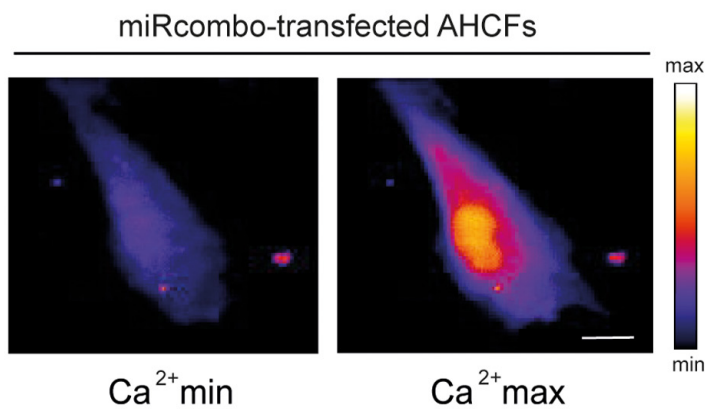

B

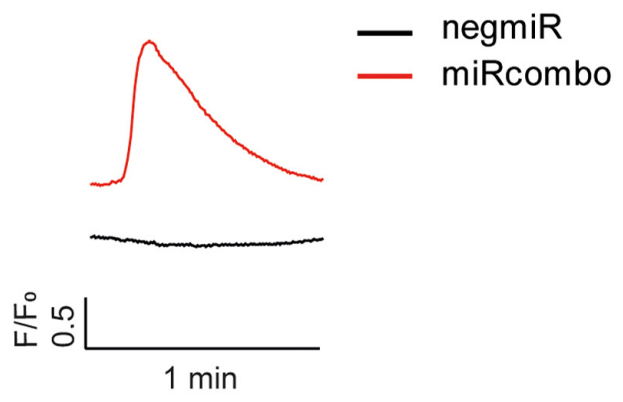

C

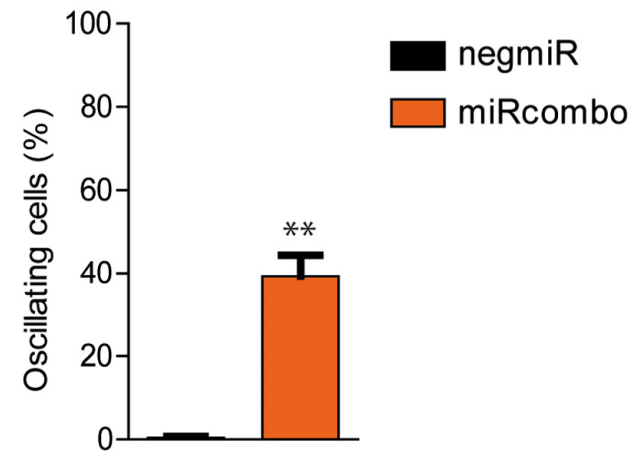

FIGURE 4 | miRcombo-transfected cells exhibit calcium transients. (A,B) Representative image, recorded with fluorescence microscopy (A) and trace (B) of $\mathrm{Ca}^{+2}$ oscillations in AHCFs transfected with miRcombo $(n=3)$ and negmiR $(n=3)$ after 30 days from transfection. Scale bar: $25 \mu \mathrm{m}$. (C) Bar graph reports the percentage of cells showing $\mathrm{Ca}^{+2}$ transients after 30 days from miRcombo and negmiR transfection. Stated $P$-value is versus negmiR.

MiRcombo, a combination of four miRNA mimics (miRs1, 133, 208, and 499) has been previously demonstrated to successfully reprogram mouse neonatal fibroblasts into functional cardiomyocytes in vitro, and to regenerate cardiomyocytes in vivo after myocardial injury (Jayawardena et al., 2012, 2015). On the other hand, the effect of miRcombo transfection on human fibroblasts has never been tested. In the perspective of a future clinical application of miRcombo approach, preclinical studies should be carried out using human cells.

In this work, DharmaFECT1 was demonstrated to be an efficient agent for miRNA delivery to AHCFs (Figures 1A,B).
Moreover, miR-1 delivery to AHCFs efficiently induced knockdown expression of TWF-1 (miR-1 target in cardiac fibroblasts), which was maintained up to $48 \mathrm{~h}$ culture time.

Then, DharmaFECT1 was employed for transfection of AHCFs with miRcombo or negmiR, and cardiac gene and protein expression of transfected AHCFs was investigated at different time steps. After 7 days from transient transfection with miRcombo, AHCFs showed upregulation of early cardiac TF expression, such as GATA4, MEF2C, TBX5, HAND2, respect to negmiR transfected cells (Figure 2). Early cardiac TFs are known to work in synergy, guiding cardiac cell fate and contractile protein expression (Olson, 2006). Nuclear localization of cardiac TFs was found in chemically induced cardiomyocytes from mouse fibroblasts ( $\mathrm{Fu}$ et al., 2015). In the future, immunostaining of cardiac TFs in miRcombo-transfected cells could help in understanding their correct location in cell nuclei, ensuring their function in inducing mature cardiomyocyte marker expression.

Then, the expression of functional and structural cardiomyocyte markers in AHCFs at longer time from transfection was evaluated. Particularly, gene expression of TNNT2 was enhanced in miRcombo transfected AHCFs respect to negmiR transfected cells after 15 days from transfection (Figure 3A). These results were also confirmed by flow cytometry, showing a reprogramming efficiency - measured as percentage of $\mathrm{cTnT}^{+}$cells - of $\sim 11 \%$ (Figure $3 \mathrm{C}$ ), which was indeed higher than the value reported for miRcombo-transfected neonatal murine cardiac fibroblasts $(\sim 3 \%)$ ( $\mathrm{Li}$ et al., 2016). On the other hand, the measured reprogramming efficiency was similar to values reported in previous investigations on direct reprogramming of AHCFs into iCMs $(\sim 13 \%$ after 2 weeks reported by Nam et al., 2013).

Additionally, a reduced expression of fibroblastic markers (VIM, DDR2 and FSP-1) was measured after 15 days of culture post-transfection (Figures 3D-F).

Notably, differently from previous studies on miRcombo transfection of mouse neonatal fibroblasts (Jayawardena et al., 2012; Dal-Pra et al., 2019), miRcombo-transfected AHCFs did not show sarcomeric organization after 15 days from miRcombo transfection, suggesting an immature phenotype (data not shown). On the other hand, previous studies reported early sarcomeric structure in AHCFs transfected with cardiac TFs and miRNAs, after at least 4-5 weeks from transfection (Nam et al., 2013; Muraoka et al., 2014). Compared to neonatal fibroblasts, AHCFs are more resistant to direct reprogramming, caused by their stable epigenetic program. For this reason, in the future, longer culture times and/or additional stimulating factors will be applied to transdifferentiate AHCFs into mature iCMs.

Upon maintaining miRcombo-transfected AHCFs in culture for 30 days, $\sim 38 \%$ of total cells exhibited spontaneous calcium oscillation, suggesting cardiomyocyte-like functionality (Figures 4A-C). This is a relevant result when compared to what reported by Nam et al. (2013) who found out a cell percentage of $\sim 15 \%$ exhibiting calcium transients induced by $\mathrm{KCl}$ stimulation, after 4 weeks from transfection. Although most of the previous studies on direct cardiac reprogramming 
analyzed calcium transient through stimulation with high $\mathrm{KCl}$ concentration or caffeine (Fu et al., 2013; Nam et al., 2013), a few authors reported the analysis of spontaneous calcium oscillation of iCMs as in our case (Smith et al., 2013). In the future, induced calcium oscillation through $\mathrm{KCl}$ or caffeine stimulation will be analyzed to confirm cardiomyocyte-like functionality.

In conclusion, results demonstrated for the first time that miRcombo transfection was able to activate a phenotypic switch of AHCFs toward cardiomyocyte-like cells. Although other methods are under study to directly reprogram AHCFs into iCMs (Nam et al., 2013; Muraoka et al., 2014), the advantage of miRcombo-mediated approach derives from the possibility for non-viral transfection, overcoming the hurdles associated with viral vectors, such as high cost, difficulties in large scale production and potential mutagenesis and immunogenic behavior (Labatut and Mattheolabakis, 2018). Indeed, safety represents one key requirement for perspective application of direct reprogramming to humans. In the present study, the overexpression of defined cardiac developmental factors and mature markers was achieved by transient transfection with miRcombo through a commercially available lipidic transfection agent. AHCFs transfected with miRcombo were able to maintain the expression of specific cardiomyocyte markers at the tested time points (7 and 15 days). Future studies will be addressed to thoroughly study miRcombomediated direct reprogramming of AHCFs at longer times and under different microenvironmental stimulations, and to develop non-viral vectors for in situ transfection with miRcombo, paving the way toward a clinical translation of direct reprogramming strategies (Muraoka et al., 2014; Li et al., 2016; Paoletti et al., 2018).

\section{CONCLUSION}

This brief report provides a preliminary study showing that a combination of four microRNAs (miRcombo) is able to drive a significant change in human fibroblast phenotype, starting their trans-differentiation toward cardiomyocyte-like cells. Despite further investigations are required to confirm and to enhance the functional activity of iCMs, the present work demonstrates that

\section{REFERENCES}

Bektik, E., and Fu, J. (2019). Ameliorating the fibrotic remodeling of the heart through direct cardiac reprogramming. Cells 8:679. doi: 10.3390/cells8070679

Chen, Y., Yang, Z., Zhao, Z.-A., and Shen, Z. (2017). Direct reprogramming of fibroblasts into cardiomyocytes. Stem Cell Res. Ther. 8:118. doi: 10.1186/s13287017-0569-3

Dal-Pra, S., Hodgkinson, C. P., and Dzau, V. J. (2019). Induced cardiomyocyte maturation: cardiac transcription factors are necessary but not sufficient. PLoS One 14:e0223842. doi: 10.1371/journal.pone. 0223842

Fu, J. D., Stone, N. R., Liu, L., and Spencer, C. I (2013). Direct reprogramming of human fibroblasts toward a cardiomyocyte-like state. Stem Cell Rep. 1, 235-247. doi: 10.1016/j.stemcr.2013.07.005

Fu, Y., Huang, C., Xu, X., Gu, H., Ye, Y., Jiang, C., et al. (2015). Direct reprogramming of mouse fibroblasts into cardiomyocytes with chemical cocktails. Cell Res. 25, 1013-1024. doi: 10.1038/cr.2015.99
miRcombo transfection is effective in directly reprogramming AHCFs into cardiomyocyte-like cells with potentialities for future clinical application.

\section{DATA AVAILABILITY STATEMENT}

The datasets generated for this study are available on request to the corresponding author.

\section{AUTHOR CONTRIBUTIONS}

CP designed the experimental part of the project, conducted experiments, and analyzed the data. CD supported CP in the in vitro culture experiments and trained $\mathrm{CP}$ in ddPCR analysis at INRIM biology lab. GT supported CP in ddPCR analysis for miR-1 overexpression and mRNA target downregulation. FD and DN supported CP in the in vitro culture experiments with adult human fibroblasts. VC supervised the work and acquired funding. The manuscript was written through main contributions by $\mathrm{CP}$ and $\mathrm{VC}$ and further help by all the authors. All authors have given approval to the final version of the manuscript.

\section{FUNDING}

The project has received funding from the European Research Council (ERC) under the European Union's Horizon 2020 Research and Innovation Programe (Grant Agreement No. 772168).

\section{ACKNOWLEDGMENTS}

The authors acknowledge support from the European Research Council (ERC) under the European Union's Horizon 2020 Research and Innovation Programe (Grant Agreement No. 772168), through BIORECAR ERC Consolidator project (www. biorecar.polito.it).

Gray, G., Toor, I., Castellan, R., Crisan, M., and Meloni, M. (2018). Resident cells of the myocardium: more than spectators in cardiac injury, repair and regeneration. Curr. Opin. Physiol. 1, 46-51. doi: 10.1016/J.COPHYS.2017.08. 001

Hashimoto, H., Olson, E. N., and Bassel-Duby, R. (2018). Therapeutic approaches for cardiac regeneration and repair. Nat. Rev. Cardiol. 15, 585-600. doi: 10.1038/ s41569-018-0036-6

He, L., and Hannon, G. J. (2004). MicroRNAs: small RNAs with a big role in gene regulation. Nat. Rev. Genet. 5, 522-531. doi: 10.1038/nrg1379

Ieda, M., Fu, J.-D., Delgado-Olguin, P., Vedantham, V., Hayashi, Y., Bruneau, B. G., et al. (2010). Direct reprogramming of fibroblasts into functional cardiomyocytes by defined factors. Cell 142, 375-386. doi: 10.1016/j.cell.2010. 07.002

Jayawardena, T. M., Egemnazarov, B., Finch, E. A., Zhang, L., Payne, J. A., Pandya, K., et al. (2012). MicroRNA-mediated in vitro and in vivo direct reprogramming of cardiac fibroblasts to cardiomyocytes. Circ. Res. 110, 1465-1473. doi: 10. 1161/CIRCRESAHA.112.269035 
Jayawardena, T. M., Finch, E. A., Zhang, L., Zhang, H., Hodgkinson, C. P., Pratt, R. E., et al. (2015). MicroRNA induced cardiac reprogramming in vivo. Circ. Res. 116, 418-424. doi: 10.1161/CIRCRESAHA.116.304510

Kitchener, D. W., Shijun, H., Shivkumar, V., Ji-Dong, F., and Ning, S. (2010). Dynamic MicroRNA expression programs during cardiac differentiation of human embryonic stem cells role for miR-499. Circ. Cardiovasc. Genet. 3, 426-435. doi: 10.1161/CIRCGENETICS.109.934281

Labatut, A. E., and Mattheolabakis, G. (2018). Non-viral based miR delivery and recent developments. Eur. J. Pharm. Biopharm. 128, 82-90. doi: 10.1016/j.ejpb. 2018.04.018

Lee, S. W. L., Paoletti, C., Campisi, M., Osaki, T., Adriani, G., Kamm, R. D., et al. (2019). MicroRNA delivery through nanoparticles. J. Control. Release 313, 80-95. doi: 10.1016/j.jconrel.2019.10.007

Li, Y., Dal-Pra, S., Mirotsou, M., Jayawardena, T. M., Hodgkinson, C. P., Bursac, N., et al. (2016). Tissue-engineered 3-dimensional (3D) microenvironment enhances the direct reprogramming of fibroblasts into cardiomyocytes by microRNAs. Sci. Rep. 6:38815. doi: 10.1038/srep38815

Muraoka, N., Yamakawa, H., Miyamoto, K., Sadahiro, T., Umei, T., Isomi, M., et al. (2014). MiR-133 promotes cardiac reprogramming by directly repressing Snail and silencing fibroblast signatures. EMBO J. 33, 1565-1581. doi: 10.15252/embj. 201387605

Nam, Y.-J., Song, K., Luo, X., Daniel, E., Lambeth, K., West, K., et al. (2013). Reprogramming of human fibroblasts toward a cardiac fate. Proc. Natl. Acad. Sci. U.S.A. 110, 5588-5593. doi: 10.1073/pnas.1301019110

Olson, E. N. (2006). Gene regulatory networks in the evolution and development of the heart. Science 313, 1922-1927. doi: 10.1126/science.1132292

Paoletti, C., Divieto, C., and Chiono, V. (2018). Impact of biomaterials on differentiation and reprogramming approaches for the generation of functional cardiomyocytes. Cells 7:114. doi: 10.3390/cells7090114

Peng, B., Chen, Y., and Leong, K. W. (2015). MicroRNA delivery for regenerative medicine. Adv. Drug Deliv. Rev. 88, 108-122. doi: 10.1016/J.ADDR.2015. 05.014

Smith, A. W., Hoyne, J. D., and Nguyen, P. K. (2013). Direct reprogramming of mouse fibroblasts to cardiomyocyte-like cells using Yamanaka factors on engineered poly(ethylene glycol) (PEG) hydrogels. Biomaterials 34, 6559-6571. doi: 10.1016/j.biomaterials.2013.05.050
Song, K., Nam, Y.-J., Luo, X., Qi, X., Tan, W., Huang, G. N., et al. (2012). Heart repair by reprogramming non-myocytes with cardiac transcription factors. Nature 485, 599-604. doi: 10.1038/nature11139

Talkhabi, M., Zonooz, E., and Baharvand, H. (2017). Boosters and barriers for direct cardiac reprogramming. Life Sci. 178, 70-86. doi: 10.1016/J.LFS.2017.04. 013

Talman, V., and Ruskoaho, H. (2016). Cardiac fibrosis in myocardial infarctionfrom repair and remodeling to regeneration. Cell Tissue Res. 365, 563-581. doi: 10.1007/s00441-016-2431-9

Tian, J., An, X., and Niu, L. (2017). Role of microRNAs in cardiac development and disease. Exp. Ther. Med. 13, 3-8. doi: 10.3892/etm.2016. 3932

van den Borne, S. W. M., Diez, J., Blankesteijn, W. M., Verjans, J., Hofstra, L., and Narula, J. (2010). Myocardial remodeling after infarction: the role of myofibroblasts. Nat. Rev. Cardiol. 7, 30-37. doi: 10.1038/nrcardio. 2009.199

van Rooij, E., Quiat, D., Johnson, B. A., Sutherland, L. B., Qi, X., Richardson, J. A., et al. (2009). A family of microRNAs encoded by myosin genes governs myosin expression and muscle performance. Dev. Cell 17, 662-673. doi: 10. 1016/j.devcel.2009.10.013

Xu, C., Lu, Y., Pan, Z., Chu, W., Luo, X., Lin, H., et al. (2011). The musclespecific microRNAs miR-1 and miR-133 produce opposing effects on apoptosis by targeting HSP60, HSP70 and caspase-9 in cardiomyocytes. J. Cell Sci. 124, 3187. doi: 10.1242/jcs.098830

Conflict of Interest: The authors declare that the research was conducted in the absence of any commercial or financial relationships that could be construed as a potential conflict of interest.

Copyright (C) 2020 Paoletti, Divieto, Tarricone, Di Meglio, Nurzynska and Chiono. This is an open-access article distributed under the terms of the Creative Commons Attribution License (CC BY). The use, distribution or reproduction in other forums is permitted, provided the original author(s) and the copyright owner(s) are credited and that the original publication in this journal is cited, in accordance with accepted academic practice. No use, distribution or reproduction is permitted which does not comply with these terms. 